\title{
Properties of Optimal Smooth Functions in Additive Models for Hedging Multivariate Derivatives*
}

\author{
Yuji Yamada \\ Graduate School of Business Sciences, University of Tsukuba \\ 3-29-1 Otsuka, Bunkyo-ku, Tokyo, Japan 112-0012 \\ E-mail: yuji@gssm.otsuka.tsukuba.ac.jp
}

\begin{abstract}
In this paper, we consider an optimal hedging problem for multivariate derivative based on the addtive sum of smooth functions on individual assets that minimize the mean square error (or the variance with zero expected value) from the derivative payoff. By applying the necessary and sufficient condition with suitable discretization, we derive a set of linear equations to construct optimal smooth functions, where we show that the computations involving conditional expectations for the multivariate derivatives may be reduced to those of unconditional expectations, and thus, the total procedure can be executed efficiently. We investigate the theoretical properties for the optimal smooth functions and clarify the following three facts: (i) the value of each individual option takes an optimal trajectory to minize the mean square hedging error under the risk neutral probability measure, (ii) optimal smooth functions for the put option may be constructed using those for the call option (and vice versa), and (iii) delta in the replicating portfolio may be computed efficietnly. Numerical experiments are included to show the effectiveness of our proposed methodology.
\end{abstract}

Keywords: Additive models, Minimum variance hedging, Basket options, Multivariate derivatives, Smooth functions

\section{Introduction}

At the heart of hedging theory is how to construct a portfolio that replicates (or approximates) the value of target asset as close as possible. In this paper, we formulate a problem of hedging multivariate derivatives based on the idea from non-parametric regression technique known as the generalized additive model (GAM; see Hastie and Tibshirani (1990), Wood (2006)), where the value of multivariate derivative is approximated by optimizing smooth functions on individual derivatives to minimize the mean square error of the terminal payoffs. Note that GAM applies cubic splines that minimizes the penalized residual sum of squares (PRSS) to fit given sample data and may be considered as the generalization of linear regression model to the additive model using the sum of smooth functions. For empirical analysis of hedging problem, GAM has been used to investigate the hedge effect of weather derivatives in Yamada (2007, 2008a, 2008b).

An application of nonparametric regression to derivative pricing/hedging problems is a well examined area in finance, in particular in the context of general nonparametric formula for option prices and/or implied volatility

${ }^{*}$ To appear in Asia-Pacific Financial Markets (DOI 10.1007/s10690-011-9145-5). Submitted: 30 April 2011. Accepted: 7 August 2011. This work is supported by Grant-in-Aid for Scientific Research (C) 22510138 from Japan Society for the Promotion of Science. 
surface. For estimating the pricing formula, Hutchinson et al. (1994) applied a nonparametric approach via learning networks given empirical observation, and since then, a number of researchers have investigated option prices using nonparametric techniques based on, e.g., neural networks (Garciaa and R. Gencay, 2000), kernel regressions (Ait-Sahalia and Lo (1998), Broadie et al. (2000)), and the so-called canonical valuation (Alcock and Gray (2005), Gray et al. (2007), Stutzer (1996); see also Daglish (2003) and references therein for a comparison of these nonparametric techniques). Note that the focus of our research is slightly different from these nonparametric approaches, in the sense that the replication of payoff for a derivative security is a main concern based on derivatives on individual assets. Also, it should be mentioned that we take a theoretical approach in a continuous time framework instead of empirical one by solving a necessarily and sufficient condition for the minimum mean square error.

For hedging European type basket options using options on individual assets, a super-hedging strategy consisting of the weighted sum of options with the same types (i.e., calls or puts) may be available as described in Hobson et al. (2005) and Su (2008), where the super-hedging strategy is to find a portfolio whose terminal value is always larger than that of the multivariate option. In the simplest case, the super-hedging strategy for basket option may be constructed by Jensen's inequality, i.e., for a convex function $g$ and $p_{i}, i=1, \ldots, m$ such that

$$
\sum_{i=1}^{m} p_{i}=1, \quad p_{i} \geq 0,
$$

we have

$$
\sum_{i=1}^{m} p_{i} g\left(x_{i}\right) \geq g\left(\sum_{i=1}^{m} p_{i} x_{i}\right), \quad{ }^{\forall} x_{i} \in \Re, i=1, \ldots, m .
$$

In the above formulation, we can regard $g$ as a payoff function, $x_{i}$ the terminal value of asset $i \in[1, m]$, and $p_{i}$ a weight parameter on asset $i$, and hence, an upper bound of European type basket option may be obtained by the weighted sum of individual options with the same payoff function $g$. If $g$ is a payoff function of call options, then the upper bound may be improved so that the super-hedging portfolio is given by the weighted sum of call options on individual assets with different values of strikes (Hobson et al. (2005), Su (2008)). Also, note that, for hedging basket options using dynamic trading strategy, a semi-definite programming based receding horizon control approach has been developed in Primbs (2009), where the problem for a European call option is formulated as a finite horizon constrained stochastic control problem.

In the current paper, we consider an optimal hedging problem for multivariate derivatives based on the additive sum of smooth functions on individual assets, namely the additive models. Note that the problem may be interpreted as an optimal approximation for the multivariate derivative using individual options with any payoff functions that minimize the mean square error (or the variance with zero expected value). By applying the necessary and sufficient condition with suitable discretization, we derive a set of linear equations to construct optimal smooth functions, where we show that the computations involving conditional expectations may be reduced to those of unconditional expectations for the multivariate derivatives, and thus, the total procedure can be executed efficiently. We investigate the theoretical properties for the optimal smooth functions and clarify the following three facts: (i) the value of each individual option takes an optimal trajectory to minimize the mean square hedging error under the risk neutral probability measure, (ii) optimal smooth functions for the put option may be constructed using those for the call option (and vice versa), and (iii) delta in the replicating portfolio may be computed efficiently. We also compare our proposed methodology with the super-hedging strategy based on the numerical experiment, and conclude that the optimal hedging strategy is better if we take standard deviation as a performance measure of the hedge, whereas in terms of the worst case error, super-hedging tends to provide a better bound with a given confidence level.

The rest of this paper is organized as follows: In Section 2, we formulate the minimum variance hedging 
problem using smooth functions, provide a necessarily and sufficient condition for the optimizers, and discuss some properties of the problem. We also show that the optimal smooth functions are found by solving a system of linear equations based on suitable discretization. In Section 3, we introduce price dynamics that enable us to replicate the terminal payoffs via dynamic trading strategy, and demonstrate how to construct the linear equations for the univariate and the multivariate cases. In Section 4, we investigate the minimum variance hedging problem under the risk neutral probability measure. In Section 5, we discuss theoretical properties of the optimal smooth functions based on the put-call parity and sensitivity analysis. Numerical experiments are also provided to illustrate our proposed methodology in Section 6. Section 7 offers some concluding remarks.

\section{Problem formulation and optimality condition}

\subsection{Minimum variance hedging problem based on the additive model}

Let $S_{i, t}, i=1, \ldots, m_{0}$ be the values of assets at time $t \in[0, T]$ under a probability space $(\Omega, \mathcal{F}, \mathbb{P})$ and filtration $\left\{\mathcal{F}_{t}\right\}_{t \in[0, T]}$. In this paper, we consider the following problem of hedging the terminal payoff of derivative security using the additive sum of smooth functions of $S_{i, t}, i=1, \ldots, m$, namely the additive model:

$$
\min _{f_{i} \in \mathcal{S}} \mathbb{E}\left[\left\{G_{T}-\sum_{i=1}^{m} f_{i}\left(S_{i, T}\right)\right\}^{2}\right],
$$

where $m \leq m_{0}$, and $G_{t}, t \in[0, T]$ stands for the value of derivative security whose terminal payoff at the maturity $T$ is a function of $m_{0}$ assets, $S_{1, T}, \ldots, S_{m_{0}, T}$.

Note that $G_{T}$ may be the terminal payoff of a illiquid (or nontraded) asset derivative. For example, if we assume that $S_{m_{0}, t}$ is nontraded until the maturity $T$ and $G_{T}$ is a function of $S_{m_{0}, T}$ with $m<m_{0}$, then the problem is to find optimal payoff functions of $m$ tradable assets to minimize the terminal hedging error from the value of illiquid asset derivative. Another case is that $G_{T}$ may be the terminal payoff of portfolio of options (such as bull or bear spreads of different underlyings) or a compound option (derivative of options). Also, in the case of basket options, $G_{T}$ may be given as

$$
G_{T}=g\left(\sum_{i=1}^{m_{0}} \alpha_{i} S_{i, T}\right),
$$

with given weight parameters $\alpha_{i}, i=1, \ldots, m_{0}$ and a payoff function $g$.

For finding optimal smooth functions of problem (1), the following Lemma is key in this paper, which is introduced in Chapter 5 of Hastie and Tibshirani (1990):

Lemma 1 Smooth functions $f_{1}^{*}, \ldots, f_{m}^{*}$ provide minimizers of problem (1), if and only if the following conditions are satisfied:

$$
\sum_{j=1}^{m} \mathbb{E}\left[f_{j}^{*}\left(S_{j, T}\right) \mid S_{i, T}\right]=\mathbb{E}\left[G_{T} \mid S_{i, T}\right], \quad i=1, \ldots, m
$$

By taking unconditional expectation for both sides in the above equation, we have

$$
\mathbb{E}\left[G_{T}\right]=\sum_{i=1}^{m} \mathbb{E}\left[f_{i}^{*}\left(S_{i, T}\right)\right] .
$$

Therefore, it holds that

$$
\operatorname{Var}\left[G_{T}-\sum_{i=1}^{m} f_{i}^{*}\left(S_{i, T}\right)\right]=\mathbb{E}\left[\left(G_{T}-\sum_{i=1}^{m} f_{i}^{*}\left(S_{i, T}\right)\right)^{2}\right] .
$$


Conditions (3) and (4) suggest that minimizing the mean square error corresponds to the variance minimization with zero mean constraint.

Before showing the solution method for smooth functions satisfying Lemma 1, we discuss how to replicate $f_{i}^{*}\left(S_{i, T}\right), i=1, \ldots, m$ as cash values. Since each $f_{i}^{*}(i=1, \ldots, m)$ is a smooth function, there are two approaches to attain $f_{i}^{*}\left(S_{i, T}\right), i=1, \ldots, m$. The first approach is to use European type calls and puts with maturity $T$ and any strikes as given by Carr and Madan (2001), where any twice continuously differentiable function, $f(x)$, of the terminal stock price $S_{T}=x$, can be replicated by a unique initial position of $f\left(S_{0}\right)-$ $f^{\prime}\left(S_{0}\right) S_{0}$ unit discount bounds, $f^{\prime}\left(S_{0}\right)$ shares, and $f^{\prime \prime}(K) \mathrm{d} K$ out-of-the money options of all strikes $K$ based on the the following relation:

$$
f(x)=\left[f\left(S_{0}\right)-f^{\prime}\left(S_{0}\right) S_{0}\right]+f^{\prime}\left(S_{0}\right) x+\int_{0}^{S_{0}} f^{\prime \prime}(K)(K-x)^{+} \mathrm{d} K+\int_{S_{0}}^{\infty} f^{\prime \prime}(K)(x-K)^{+} \mathrm{d} K
$$

The advantage of this approach is that we do not have to estimate any parameters such as volatilities or mean rates of returns of the underlying assets once the target payoff function $f$ is specified.

The second approach is to dynamically trade $S_{i, t}$ to replicate the terminal payoff $f_{i}^{*}\left(S_{i, T}\right)$, similar to the Black-Scholes-Merton dynamic hedging strategy (Black and Scholes (1973), Merton (1973)). For this approach to be applicable, we need to introduce price dynamics for $S_{i, t}, i=1, \ldots, m$, namely the "dynamic hedging model." We will further discuss this approach in Section 3.

\subsection{Solution method for optimal smooth functions}

Recall that, from Lemma 1, we need to find a set of functions, $f_{1}^{*}, \ldots, f_{m}^{*}$, satisfying (2) to solve the minimum variance hedging problem in (1). Noting that $G_{T}$ is nonnegative in general, there exists a function $\hat{g}_{i}$ such that

$$
\mathbb{E}\left[G_{T} \mid S_{i, T}\right]=\hat{g}_{i}\left(S_{i, T}\right)
$$

for each $i=1, \ldots, m$ (See pp. 81 in Shreve (2004)).

Assume that each pair $\left(S_{i, T}, S_{j, T}\right), i, j=1, \ldots, m, i \neq j$ has a joint probability density function (PDF) given by $\phi_{S_{i}, S_{j}}\left(x_{i}, x_{j}\right)$, and define the following conditional PDF:

$$
\phi_{S_{j} \mid S_{i}}\left(x_{j} \mid x_{i}\right):=\frac{\phi_{S_{i}, S_{j}}\left(x_{i}, x_{j}\right)}{\phi_{S_{i}}\left(x_{i}\right)},
$$

where $\phi_{S_{i}}\left(x_{i}\right)$ is the marginal PDF of $S_{i, T}, i=1, \ldots, m$. Then, for given $f_{i}^{*}, i=1, \ldots, m$, there exist functions $\hat{f}_{i} i=1, \ldots, m$ such that

$$
\mathbb{E}\left[f_{j}^{*}\left(S_{j, T}\right) \mid S_{i, T}\right]=\hat{f}_{i}\left(S_{i, T}\right), \quad i=1, \ldots, m .
$$

where each $\hat{f}_{i}$ may be written as

$$
\hat{f}_{i}\left(x_{i}\right)=\int_{\Re} f_{j}^{*}\left(x_{j}\right) \cdot \phi_{S_{j} \mid S_{i}}\left(x_{j} \mid x_{i}\right) \mathrm{d} x_{j}, \quad x_{i} \in \Re .
$$

Therefore, the problem reduces to finding a set of real-valued functions, $f_{1}^{*}, \ldots, f_{m}^{*}$, satisfying the following system of equations:

$$
f_{i}^{*}\left(x_{i}\right)+\sum_{j \neq i} \int_{\Re} f_{j}^{*}\left(x_{j}\right) \cdot \phi_{S_{j} \mid S_{i}}\left(x_{j} \mid x_{i}\right) \mathrm{d} x_{j}=\hat{g}_{i}\left(x_{i}\right), \quad i=1, \ldots, m
$$

Note that $\hat{g}_{i}, i=1, \ldots, m$ in (6) may be defined explicitly in the following sections, and so far, we assume that these functions are given.

We would like to find $f_{i}^{*}, i=1, \ldots, m$ such that (6) holds for appropriate domains of input variables, $x_{i}, i=1, \ldots, m$. Here we provide a solution method consisting of the following three steps: 
1. Discretize condition (6) for $y, x_{i}$ and $x_{j}(i, j=1, \ldots, m)$ dimensions to obtain a set of linear equations.

2. Solve the set of linear equations to find discretized points of smooth functions.

3. Construct smooth functions using cubic splines.

Note that the above method may be applied if joint PDFs of pairs in $\left(S_{i, T}, S_{j, T}\right), i, j=1, \ldots, m, i \neq j$ are given.

First, we discretize condition (6) to approximate the integrals as

$$
f_{i}^{*}\left(x_{i}\right)+\sum_{j \neq i} \sum_{l=1}^{N}\left[f_{j}^{*}\left(x_{j}^{(l)}\right) \cdot \phi_{S_{j} \mid S_{i}}\left(x_{j}^{(l)} \mid x_{i}\right) \delta_{j}\right]=\hat{g}_{i}\left(x_{i}\right), \quad i=1, \ldots, m
$$

for given $x_{i}, i=1, \ldots, m$, where $\delta_{j}$ is assumed to satisfy

$$
\sum_{l=1}^{N} \phi_{S_{j} \mid S_{i}}\left(x_{j}^{(l)} \mid x_{i}\right) \delta_{j}=1, \quad j \neq i
$$

Note that $\delta_{j}$ may depend on $x_{i}$ as well, but we will omit to specify that dependence for brevity. We then discretize condition (7) for $x_{i}$ dimensions, e.g., $x_{i}^{(k)}, k=1, \ldots, N$, as

$$
f_{i}^{*}\left(x_{i}^{(k)}\right)+\sum_{j \neq i} \sum_{l=1}^{N}\left[f_{j}^{*}\left(x_{j}^{(l)}\right) \cdot \phi_{S_{j} \mid S_{i}}\left(x_{j}^{(l)} \mid x_{i}^{(k)}\right) \delta_{j}\right]=\hat{g}_{i}\left(x_{i}^{(k)}\right), \quad i=1, \ldots, m .
$$

Let $\boldsymbol{f}_{i} \in \Re^{N}(i=1, \ldots, m)$ and $\boldsymbol{g}_{i} \in \Re^{N}$ be real-valued vectors whose $k$-th entries are, respectively, given as

$$
\boldsymbol{f}_{i}[k]=f_{i}^{(k)}:=f_{i}^{*}\left(x_{i}^{(k)}\right), \quad \boldsymbol{g}_{i}[k]=\hat{g}_{i}\left(x_{i}^{(k)}\right), \quad k=1, \ldots, N .
$$

Also, let $\Phi_{i, j} \in \Re^{N \times N}(i, j=1, \ldots, m, i \neq j)$ be matrices whose $(k, l)$-entries are given as

$$
\Phi_{i, j}[k, l]:=\phi_{S_{j} \mid S_{i}}\left(x_{j}^{(l)} \mid x_{i}^{(k)}\right) \delta_{j}, \quad k, l=1, \ldots, N
$$

With these definitions and notations, we have the following proposition:

Proposition 1 For each $i=1, \ldots, m$, condition (6) may be discretized as

$$
\boldsymbol{f}_{i}+\sum_{j \neq i} \Phi_{i, j} \boldsymbol{f}_{j}=\boldsymbol{g}_{i}
$$

Consequently, we obtain the following system of linear equations for $\boldsymbol{f}:=\left[\boldsymbol{f}_{1}^{\top}, \ldots, \boldsymbol{f}_{m}^{\top}\right]^{\top} \in \Re^{m N}$ :

$$
\Phi \boldsymbol{f}=\boldsymbol{g}
$$

where

$$
\Phi:=\left[\begin{array}{ccccc}
I_{N \times N} & \Phi_{1,2} & \Phi_{1,3} & \cdots & \Phi_{1, m} \\
\Phi_{2,1} & I_{N \times N} & \Phi_{2,3} & \cdots & \Phi_{2, m} \\
\Phi_{3,1} & \Phi_{3,2} & I_{N \times N} & \ddots & \Phi_{3, m} \\
\vdots & \vdots & \ddots & \ddots & \vdots \\
\Phi_{m, 1} & \Phi_{m, 2} & \Phi_{m, 3} & \cdots & I_{N \times N}
\end{array}\right] \in \Re^{m N \times m N}, \quad \boldsymbol{g}:=\left[\begin{array}{c}
\boldsymbol{g}_{1} \\
\boldsymbol{g}_{2} \\
\vdots \\
\boldsymbol{g}_{m}
\end{array}\right] \in \Re^{m N}
$$


Although the solution to (9) may not be unique, it can be expressed using the generalized inverse matrix as

$$
\boldsymbol{f}=\Phi^{\top}\left[\Phi \Phi^{\top}\right]^{-1} \boldsymbol{g}
$$

Then, the optimal smooth functions, $f_{i}^{*}, i=1, \ldots, m$, may be constructed using cubic splines,

$$
f_{i}^{*}(x)=c_{0}+c_{1} x+\frac{1}{12} \sum_{k=1}^{N} \theta_{k}\left|x-x_{i}^{(k)}\right|^{3}
$$

where $c_{0}, c_{1}$ and $\theta_{k}, k=1, \ldots, N$ are found to satisfy $f_{i}^{*}\left(x_{i}^{(k)}\right)=f_{i}^{(k)}$ and

$$
\sum_{k=1}^{N} \theta_{k}=0, \quad \sum_{k=1}^{N} \theta_{k} x_{i}^{(k)}=0 .
$$

\section{Construction of optimal smooth functions}

In this section, we introduce price dynamics for $S_{i, t}$ that enable us to replicate the terminal payoff $f_{i}^{*}\left(S_{i, T}\right)$ using dynamic trading strategy, and demonstrate how to specify $\hat{g}_{i}$ in (6). Assume that, under the probability space $(\Omega, \mathcal{F}, \mathbb{P})$, the price dynamics of $S_{1, t}, \ldots, S_{m, t}$ are governed by the following stochastic differential equations (SDEs),

$$
\mathrm{d} S_{i, t}=\mu_{i} S_{i, t} \mathrm{~d} t+\sigma_{i} S_{i, t} \mathrm{~d} W_{i, t}, \quad i=1, \ldots, m_{0},
$$

where $W_{1, t}, \ldots, W_{m_{0}, t}$ are correlated Brownian motions with $\mathrm{d} W_{i, t} \mathrm{~d} W_{j, t}=\rho_{i j} \mathrm{~d} t, i, j=1, \ldots, m_{0}, i \neq j$.

Remark 1 One of the advantages for considering (12) is that there exists a dynamic trading strategy to replicate the terminal payoff $f_{i}^{*}\left(S_{i, T}\right)$ once the optimal smooth functions are specified. Let $\Delta_{i, t}^{*}$ be shares of $S_{i, t}$ possessed at time $t$ in the replicating portfolio. Then, $\Delta_{i, t}^{*}$ may be obtained as $\Delta_{i, t}^{*}=\partial V_{i, t} / \partial S_{i, t}$ with $V_{i, t}:=e^{-r(T-t)} \tilde{\mathbb{E}}\left[f_{i}^{*}\left(S_{i, T}\right) \mid S_{i, t}\right]$. Here $\tilde{\mathbb{E}}$ is the expectation under a risk neutral probability measure $\tilde{\mathbb{P}}$ (being equivalent to $\mathbb{P})$. Although $\tilde{\mathbb{P}}$ is not unique in general due to the incompleteness of the market, the risk neutral process of $S_{i, t}$ may be described as

$$
\mathrm{d} S_{i, t}=r S_{i, t} \mathrm{~d} t+\sigma_{i} S_{i, t} \mathrm{~d} \tilde{W}_{i, t}, \quad i=1, \ldots, m,
$$

where $\tilde{W}_{i, t}$ is a Brownian motion under the corresponding risk neutral probability measure $\tilde{\mathbb{P}}$. Note that $V_{i, t}$ also provides the value of the self-financing portfolio at time $t \in[0, T]$ and that the trading strategy $\Delta_{i, t}^{*}$ is independent of the choice of risk neutral probability measure.

\subsection{Hedging of illiquid asset derivatives}

First, we demonstrate the case where $G_{T}$ depends on the terminal value of a single asset, illustrating the problem of hedging derivative security whose underlying is nontraded (or illiquid) using derivatives on liquidly traded assets. Let $m_{0}:=m+1$ in (12), and suppose that the $(m+1)$-th asset, $S_{m_{0}, t}$, is nontraded (or illiquid) until the maturity $T$ and that the other $m$ assets can be traded liquidly. ${ }^{1}$ To distinguish the notation between the tradable and nontradable assets, we use $Y_{t}$ for the value of nontraded asset, i.e., $Y_{t} \equiv S_{m_{0}, t}$ is governed by the following SDE:

$$
\mathrm{d} Y_{t}=\mu_{m+1} Y_{t} \mathrm{~d} t+\sigma_{m+1} Y_{t} \mathrm{~d} W_{m+1, t} .
$$

\footnotetext{
${ }^{1}$ The problem setting in this section addresses the one in Schwartz and Tebaldi (2006) when $m=1$. Also, the problem in this case is closely related to the pioneering work of Duffie and Richardson (1991) for hedging the spot price using the self-financing portfolio of future price. Note that, in our formulation, we intend to hedge the payoff of illiquid asset derivatives using liquidly traded asset derivatives with any payoff functions.
} 
Also, let the terminal value of $G_{t}$ satisfy

$$
G_{T}=g\left(Y_{T}\right),
$$

where $g$ is a given payoff function. For simplicity, $\mu_{i}, \sigma_{i}$ and $\rho_{i j}(i, j=1, \ldots, m+1, i \neq j)$ are assumed to be constant, although the result can readily be generalized for the case with deterministic functions of $t$. In this case, $Y_{T}$ and $S_{i, T}, i=1, \ldots, m$ are given as

$$
Y_{T}=Y_{0} e^{\nu_{m+1} T+\sigma_{m+1} W_{m+1, T}}, \quad S_{i, T}=S_{i, 0} e^{\nu_{i} T+\sigma_{i} W_{i, T}},
$$

where

$$
\nu_{i}:=\mu_{i}-\frac{\sigma_{i}^{2}}{2}, i=1, \ldots, m+1 .
$$

With these definitions, we would like to characterize the conditional expectations in (2). Since the information content in $S_{i, t}$ is the same as that in $W_{i, t}$, condition (2) may be rewritten as

$$
\sum_{j=1}^{m} \mathbb{E}\left[f_{j}^{*}\left(S_{j, T}\right) \mid W_{i, T}\right]=\mathbb{E}\left[g\left(Y_{T}\right) \mid W_{i, T}\right], \quad i=1, \ldots, m .
$$

Let $p_{j \mid i}\left(w_{j} \mid w_{i}\right), j \neq i$ be the conditional $\mathrm{PDF}$ of $W_{j, t}$ given $W_{i, t}$, i.e.,

$$
p_{j \mid i}\left(w_{j} \mid w_{i}\right):=\frac{1}{\sqrt{2 \pi\left(1-\rho_{i j}^{2}\right) T}} \exp \left\{-\frac{\left(w_{j}-\rho_{i j} w_{i}\right)^{2}}{2\left(1-\rho_{i j}^{2}\right) T}\right\} .
$$

Based on the same argument as that for condition (6), the problem boils down to searching for smooth functions, $f_{i}^{*}, i=1, \ldots, m$, such that

$$
f_{i}^{*}\left(S_{i, 0} e^{\nu_{i} T+\sigma_{i} w_{i}}\right)+\sum_{j \neq i} \int_{-\infty}^{\infty} f_{j}^{*}\left(S_{j, 0} e^{\nu_{j} T+\sigma_{j} w_{j}}\right) p_{j \mid i}\left(w_{j} \mid w_{i}\right) \mathrm{d} w_{j}=\hat{g}_{i}\left(w_{i}\right), \quad i=1, \ldots, m .
$$

Here $\hat{g}_{i}$ is a function satisfying

$$
\hat{g}_{i}\left(W_{i, T}\right)=\mathbb{E}\left[g\left(Y_{T}\right) \mid W_{i, T}\right], \quad i=1, \ldots, m .
$$

The following proposition provides an explicit formula for the function $\hat{g}_{i}$ :

Proposition 2 Smooth functions $\hat{g}_{i}, i=1, \ldots, m$ satisfying (18) may be represented as

$$
\hat{g}_{i}\left(w_{i}\right)=Y_{0} \exp \left\{\left(\mu_{m+1}-\frac{\rho_{i(m+1)}^{2} \sigma_{m+1}^{2}}{2}\right) T+\rho_{i(m+1)} \sigma_{m+1} w_{i}\right\} \mathbf{N}\left(d_{1}\left(w_{i}\right)\right)-K \mathbf{N}\left(d_{2}\left(w_{i}\right)\right)
$$

when $g(y)=(y-K)^{+}$for European call options, or

$$
\hat{g}_{i}\left(w_{i}\right)=-Y_{0} \exp \left\{\left(\mu_{m+1}-\frac{\rho_{i(m+1)}^{2} \sigma_{m+1}^{2}}{2}\right) T+\rho_{i(m+1)} \sigma_{m+1} w_{i}\right\} \mathbf{N}\left(-d_{1}\left(w_{i}\right)\right)+K \mathbf{N}\left(-d_{2}\left(w_{i}\right)\right)
$$

when $g(y)=(K-y)^{+}$for European put options, where $\mathbf{N}$ is the standard normal distribution function, and $d_{1}\left(w_{i}\right)$ and $d_{2}\left(w_{i}\right)$ are defined as

$$
\begin{aligned}
& d_{1}\left(w_{i}\right):=\frac{1}{\sigma_{m+1} \sqrt{\left(1-\rho_{i(m+1)}^{2}\right) T}}\left[\ln \left(\frac{Y_{0}}{K}\right)+\rho_{i(m+1)} \sigma_{m+1} w_{i}+\left(\mu_{m+1}+\frac{\sigma_{m+1}^{2}}{2}-\rho_{i(m+1)}^{2} \sigma_{m+1}^{2}\right) T\right], \\
& d_{2}\left(w_{i}\right):=\frac{1}{\sigma_{m+1} \sqrt{\left(1-\rho_{i(m+1)}^{2}\right) T}}\left[\ln \left(\frac{Y_{0}}{K}\right)+\rho_{i(m+1)} \sigma_{m+1} w_{i}+\left(\mu_{m+1}-\frac{\sigma_{m+1}^{2}}{2}\right) T\right] .
\end{aligned}
$$


Proof: To simplify the notation, we assume that $i=m=1$ and $m_{0}=m+1=2$, although the same result can be obtained by suitable replacement of parameters. At first, we represent the value processes (12) and (13) using independent Brownian motions instead of correlated Brownian motions. Consider the Cholesky decomposition for the covariance matrix of

$$
\left[\frac{\mathrm{d} S_{1, t}}{S_{1, t}}, \frac{\mathrm{d} Y_{t}}{Y_{t}}\right]^{\top}
$$

given as

$$
L L^{\top} \mathrm{d} t \in \Re^{2 \times 2},
$$

where

$$
L=\left[\begin{array}{cc}
\sigma_{1} & 0 \\
\rho_{12} \sigma_{2} & \sigma_{2} \sqrt{1-\rho_{12}^{2}}
\end{array}\right] .
$$

Then, there exist independent Brownian motions $B_{1, t}, B_{2, t}$ such that the value processes of $Y_{t}$ and $S_{1, t}$ have equivalent representations to equations (12) and (13) as

$$
\left[\begin{array}{c}
\mathrm{d} S_{1, t} / S_{1, t} \\
\mathrm{~d} Y_{t} / Y_{t}
\end{array}\right]=\left[\begin{array}{l}
\mu_{1} \\
\mu_{2}
\end{array}\right] \mathrm{d} t+L\left[\begin{array}{c}
\mathrm{d} B_{1, t} \\
\mathrm{~d} B_{2, t}
\end{array}\right]
$$

In this case, $Y_{T}$ and $S_{1, T}$ are given by

$$
\begin{aligned}
S_{1, T} & =S_{1,0} \exp \left\{\left(\mu_{1}-\frac{\sigma_{1}^{2}}{2}\right) T+\sigma_{1} B_{1, T}\right\}, \\
Y_{T} & =Y_{0} \exp \left\{\left(\mu_{2}-\frac{\sigma_{2}^{2}}{2}\right) T+\rho_{12} \sigma_{2} B_{1, T}+\sigma_{2} \sqrt{1-\rho_{12}^{2}} B_{2, T}\right\},
\end{aligned}
$$

where

$$
\sigma_{11}=\sigma_{1}, \sigma_{21}=\rho_{12} \sigma_{2}, \sigma_{22}=\sigma_{2} \sqrt{1-\rho_{12}^{2}}
$$

We will compute a function $\hat{g}_{1}$ satisfying $^{2}$

$$
\hat{g}_{1}\left(B_{1, T}\right)=\mathbb{E}\left[g\left(Y_{T}\right) \mid B_{1, T}\right]
$$

Since $B_{0, T}$ is independent of $B_{1, T}$, we can apply the so-called Independence Lemma (see pp. 73 in Shreve (2004)) that $\hat{g}_{1}$ is a function of a dummy variable, $w_{1}$, given by the the following unconditional expectation:

$$
\hat{g}_{1}\left(w_{1}\right)=\mathbb{E}\left[g\left(Y_{0} \exp \left\{\left(\mu_{2}-\frac{\sigma_{2}^{2}}{2}\right) T+\rho_{12} \sigma_{2} w_{1}+\sigma_{2} \sqrt{1-\rho_{12}^{2}} B_{2, T}\right\}\right)\right]
$$

Let $g(y)=(y-K)^{+}$, and $\hat{Y}_{0}, \hat{\sigma}$, and $\hat{\nu}$ be defined as

$$
\begin{aligned}
\hat{Y}_{0} & :=Y_{0} \exp \left(\rho_{12} \sigma_{2} w_{1}\right) \\
\hat{\sigma} & :=\sigma_{2} \sqrt{1-\rho_{12}^{2}} \\
\hat{\nu} & :=\mu_{2}-\frac{\sigma_{2}^{2}}{2}+\frac{\hat{\sigma}^{2}}{2}=\mu_{2}-\frac{\rho_{12}^{2}}{2} .
\end{aligned}
$$

Then, we have

$$
\hat{g}_{1}\left(w_{1}\right)=\mathbb{E}\left[\left(\hat{Y}_{0} \exp \left\{\left(\hat{\nu}-\frac{\hat{\sigma}^{2}}{2}\right) T+\hat{\sigma} B_{2, T}\right\}-K\right)^{+}\right]
$$

\footnotetext{
${ }^{2}$ We use the same notation $\hat{g}_{1}$ as that in (18) with $i=1$, because these functions are actually equivalent.
} 
The first term in $(\cdots)^{+}$of $(22)$ is a geometric Brownian motion with mean rate of return $\hat{\nu}$, volatility $\hat{\sigma}$, and an initial value $\hat{Y}_{0}$. Therefore, we can compute the right hand side of equation (22) using the Black-Scholes-Merton formula (Black and Scholes (1973), Merton (1973)) without discounting as

$$
\hat{g}_{1}\left(w_{1}\right)=e^{\hat{\nu} T} \hat{Y}_{0} \mathbf{N}\left(d_{1}\right)-K \mathbf{N}\left(d_{2}\right)
$$

to conclude that condition (19) holds, where

$$
\begin{aligned}
& d_{1}:=\frac{\ln \left(\hat{Y}_{0} / K\right)+\left(\hat{\nu}+\hat{\sigma}^{2} / 2\right) T}{\hat{\sigma} \sqrt{T}}=d_{1}\left(w_{1}\right) \\
& d_{2}:=\frac{\ln \left(\hat{Y}_{0} / K\right)+\left(\hat{\nu}-\hat{\sigma}^{2} / 2\right) T}{\hat{\sigma} \sqrt{T}}=d_{2}\left(w_{1}\right) .
\end{aligned}
$$

Similarly, we can get equation (20) for European put options.

With the similar argument to the derivation of condition (9), we can construct the following set of linear equations by discretizing $p_{j \mid i}\left(w_{j} \mid w_{i}\right), \hat{f}_{i}\left(w_{i}\right):=f_{i}^{*}\left(S_{i, 0} e^{\nu_{i} T+\sigma_{i} w_{i}}\right)$, and $\hat{g}_{i}\left(w_{i}\right)$ for $w_{i}$ and $w_{j}$ dimensions:

$$
\left[\begin{array}{cccc}
I_{N \times N} & \Phi_{1,2} & \cdots & \Phi_{1, m} \\
\Phi_{2,1} & I_{N \times N} & \cdots & \Phi_{2, m} \\
\vdots & \ddots & \ddots & \vdots \\
\Phi_{m, 1} & \Phi_{m, 2} & \cdots & I_{N \times N}
\end{array}\right]\left[\begin{array}{c}
\hat{\boldsymbol{f}}_{1} \\
\hat{\boldsymbol{f}}_{2} \\
\vdots \\
\hat{\boldsymbol{f}}_{m}
\end{array}\right]=\left[\begin{array}{c}
\hat{\boldsymbol{g}}_{1} \\
\hat{\boldsymbol{g}}_{2} \\
\vdots \\
\hat{\boldsymbol{g}}_{m}
\end{array}\right]
$$

Then, optimal smooth functions $f_{i}^{*}, i=1, \ldots, m$ are obtained using cubic splines.

\subsection{Optimal hedging of multivariate derivatives}

Next, we will consider the case of multivariate derivatives, where $G_{T}$ is given by a function of general $m_{0}$ assets, $S_{1, T}, \ldots, S_{m_{0}, T}$, i.e.,

$$
G_{T}:=g\left(S_{1, T}, \ldots, S_{m_{0}, T}\right),
$$

for a given $m_{0}$-variate function $g$, where each asset price is assumed to follow the SDEs in (12). The minimum variance hedging problem (1) is to find smooth payoff functions for terminal values of individual assets, $S_{i, T}, i=$ $1, \ldots, m$, that approximate the terminal payoff of the multivariate derivative as close as possible in the minimum mean square sense. Note that the number of individual assets, $m$, may be greater than the dimension of multivariate assets, $m_{0}$, but usually, is selected to satisfy $m \leq m_{0}$. Because it does not make any mathematical difference and the same result may be obtained by replacing $m$ with $m_{0}$ in $G_{T}$ or related variables/functions, here we assume $m=m_{0}$ for simplicity.

Recall that optimal smooth functions of $(1)$, denoted by $f_{i}^{*}\left(w_{i}\right), i=1, \ldots, m$, need to satisfy

$$
\sum_{j=1}^{m} \mathbb{E}\left[f_{j}^{*}\left(S_{j, T}\right) \mid W_{i, T}\right]=\mathbb{E}\left[G_{T} \mid W_{i, T}\right], \quad i=1, \ldots, m .
$$

or using the conditional PDFs in (16), it holds that

$$
f_{i}^{*}\left(S_{i, 0} e^{\nu_{i} T+\sigma_{i} w_{i}}\right)+\sum_{j \neq i} \int_{-\infty}^{\infty} f_{j}^{*}\left(S_{j, 0} e^{\nu_{j} T+\sigma_{j} w_{j}}\right) p_{j \mid i}\left(w_{j} \mid w_{i}\right) \mathrm{d} w_{j}=\hat{g}_{i}\left(w_{i}\right), \quad i=1, \ldots, m,
$$

with $f_{i}^{*}$ and $\hat{g}_{i}$, where $\hat{g}_{i}$ is now defined by the following conditional expectation:

$$
\hat{g}_{i}\left(W_{i, T}\right)=E\left[g\left(S_{1, T}, \ldots, S_{m, T}\right) \mid W_{i, T}\right], \quad i=1, \ldots, m .
$$

The following theorem shows that the function $\hat{g}_{i}$ may be represented using unconditional expectation and thus be computed efficiently: 
Theorem 1 For each $i \in[1, m]$ and a (nonrandom) dummy variable $w_{i} \in \Re$, there exists a function $h_{i}$ satisfying

$$
\hat{g}_{i}\left(w_{i}\right)=\mathbb{E}\left[h_{i}\left(w_{i}, Z_{1}, \ldots, Z_{m-1}\right)\right],
$$

where $Z_{1}, \ldots, Z_{m-1}$ are $(\mathcal{F}$-measurable) independent normal random variables s.t.

$$
Z_{i} \sim \mathcal{N}(0, T), i=1, \ldots, m
$$

Proof: Here we consider the case $i=1$, although the same technique may be applied for $i=2, \ldots, m$.

Let the covariance matrix of

$$
\left[\frac{\mathrm{d} S_{1, t}}{S_{1, t}}, \ldots, \frac{\mathrm{d} S_{m, t}}{S_{m, t}}\right]^{\top}
$$

be decomposed as $L L^{\top} \mathrm{d} t$, where $L$ is a lower triangular matrix defined by

$$
L:=\left[\begin{array}{cccc}
\sigma_{11} & 0 & \cdots & 0 \\
\sigma_{21} & \sigma_{22} & \ddots & \vdots \\
\vdots & \vdots & \ddots & 0 \\
\sigma_{m 1} & \sigma_{m 2} & \cdots & \sigma_{m m}
\end{array}\right] \in \Re^{m \times m}, \quad \sigma_{11}=\sigma_{1}
$$

based on the Cholesky decomposition. Then, we obtain the following equivalent representation to (12):

$$
\left[\begin{array}{c}
\mathrm{d} S_{1, t} / S_{1, t} \\
\vdots \\
\mathrm{d} S_{m, t} / S_{m, t}
\end{array}\right]=\left[\begin{array}{c}
\mu_{1} \\
\vdots \\
\mu_{m}
\end{array}\right] \mathrm{d} t+L\left[\begin{array}{c}
\mathrm{d} B_{1, t} \\
\vdots \\
\mathrm{d} B_{m, t}
\end{array}\right] .
$$

where $B_{1, t}, \ldots, B_{m, t}$ are independent Brownian motions with $B_{1, t} \equiv W_{1, t}$. Since $S_{i, T}$ is expressed as

$$
S_{i, T}=S_{i, 0} \exp \left(\nu_{i} T+\sum_{j=1}^{i} \sigma_{i j} B_{j, T}\right), \quad i=1, \ldots, m
$$

there exists a function $h_{1}$ such that

$$
g\left(S_{1, T}, \ldots, S_{m, T}\right)=h_{1}\left(W_{1, T}, B_{2, T}, \ldots, B_{m, T}\right)
$$

and we have

$$
\begin{aligned}
\mathbb{E}\left[g\left(S_{1, T}, \ldots, S_{m, T}\right) \mid S_{1, T}\right] & =\mathbb{E}\left[g\left(S_{1, T}, \ldots, S_{m, T}\right) \mid W_{1, T}\right] \\
& =\mathbb{E}\left[h_{1}\left(W_{1, T}, B_{2, T}, \ldots, B_{m, T}\right) \mid W_{1, T}\right]
\end{aligned}
$$

We further examine the conditional expectation of (29) below.

First, we note that $S_{1, T}$ is a function of $W_{1, T}$ and is independent of the other factors, $B_{2, T}, \ldots, B_{m, T}$. This indicates that there exists a sigma algebra $\mathcal{G}_{1}(\subset \mathcal{F})$ such that both $W_{1, T}$ and $S_{1, T}$ are $\mathcal{G}_{1}$-measurable and $B_{2, T}, \ldots, B_{m, T}$ are independent of $\mathcal{G}_{1}$. Then we can apply the Independence Lemma that a function $\hat{h}_{1}$ of a dummy variable $w_{1} \in \Re$,

$$
\hat{h}_{1}\left(w_{1}\right):=\mathbb{E}\left[h_{1}\left(w_{1}, B_{2, T}, \ldots, B_{m, T}\right)\right]
$$

satisfies the following condition:

$$
\begin{aligned}
\hat{h}_{1}\left(W_{1, T}\right) & =\mathbb{E}\left[h_{1}\left(W_{1, T}, B_{2, T}, \ldots, B_{m, T}\right) \mid W_{1, T}\right] \\
& =\mathbb{E}\left[g\left(S_{1, T}, \ldots, S_{m, T}\right) \mid W_{1, T}\right] \\
& =\hat{g}_{1}\left(W_{1, T}\right)
\end{aligned}
$$


Clearly, conditions (30) and (31) indicate that the statement in the theorem holds with $i=1, h_{1}$ in (28), and

$$
Z_{i} \equiv B_{i+1, T} \sim \mathcal{N}(0, T), \quad i=1, \ldots, m-1 .
$$

Similarly, we can obtain $h_{i}, i=2, \ldots, m$ by reordering $S_{1, t}, \ldots, S_{m, t}$ so that $S_{i, t}$ is the first entry when applying the Cholesky decomposition.

We see that, for any given real number $w_{i} \in \Re, i=1, \ldots, m, \hat{g}_{i}\left(w_{i}\right)$ is computed by the unconditional expectation in (26). In general, this computation involves multiple integration, but usually executed efficiently based on the Monte Carlo method by generating independent Gaussian random numbers. Note that, once a set of random numbers is generated, we can compute $\hat{g}_{i}\left(w_{i}\right)$ for different values of $w_{i}=w_{i}^{(k)}, k=1, \ldots, N$ using the same set of random numbers to construct a real-valued vector $\hat{\boldsymbol{g}}_{i} \in \Re^{N}$ in the right hand side of equation (23). Then, we solve the set of linear equations for $\hat{\boldsymbol{f}}_{i}, i=1, \ldots, m$ to find the optimal smooth functions using cubic splines. We will illustrate our proposed method based on the numerical experiment in Section 6 .

\section{Optimal smooth functions under risk neutral measure}

In the previous sections, we have discussed the minimum variance hedging problem, in which the mean square hedging error is taken under the physical probability measure $\mathbb{P}$. In this section, we take a risk neutral probability measure instead of the physical one, and consider the following problem under the risk neutral measure $\tilde{\mathbb{P}}$ with $G_{T}$ defined in (24):

$$
\min _{f_{i} \in \mathcal{S}} \tilde{\mathbb{E}}\left[\left\{G_{T}-\sum_{i=1}^{m} f_{i}\left(S_{i, T}\right)\right\}^{2}\right],
$$

where $\tilde{\mathbb{E}}$ is the expectation under $\tilde{\mathbb{P}}$.

Let $f_{i}^{*}, i=1, \ldots, m$ be optimal smooth functions of problem (32). Then, based on the similar discussion to the derivation of condition (3), it holds that

$$
\tilde{\mathbb{E}}\left[G_{T}\right]=\sum_{i=1}^{m} \tilde{\mathbb{E}}\left[f_{i}^{*}\left(S_{i, T}\right)\right]
$$

By multiplying the discount factor $e^{-r T}$ from both sides of the above equation, we have

$$
e^{-r T \tilde{\mathbb{E}}}\left[G_{T}\right]=\sum_{i=1}^{m} e^{-r T} \tilde{\mathbb{E}}\left[f_{i}^{*}\left(S_{i, T}\right)\right]
$$

where $r>0$ is the risk free interest rate. Condition (34) implies that the value of basket option equals to the sum of individual option values with payoff functions, $f_{i}^{*}, i=1, \ldots, m$. For brevity of the notation, we omit the discount factor and refer to $G_{t}$ defined by

$$
G_{t}:=\tilde{\mathbb{E}}\left[G_{T} \mid \mathcal{F}_{t}\right]
$$

as the value of basket option. Similarly,

$$
V_{i, t}:=\tilde{\mathbb{E}}\left[f_{i}^{*}\left(S_{i, T}\right) \mid \mathcal{F}_{t}\right]
$$

may be thought of the value of individual option (without discounting) at time $t \in[0, T]$ having a payoff function $f_{i}^{*}$.

Let $S_{i, t}, i=1, \ldots, m_{0}$ follow SDEs in (12). Then, from the Markov property (see, e.g., Shreve (2004)), there exists a function $h_{i, t}^{*}$ such that

$$
V_{i, t}=h_{i, t}^{*}\left(S_{i, t}\right):=\tilde{\mathbb{E}}\left[f_{i}^{*}\left(S_{i, T}\right) \mid S_{i, t}\right], \quad t \in[0, T], i=1, \ldots, m .
$$


where $h_{i, T}^{*}=f_{i}^{*}$. In this case, an interesting question is to ask if $h_{i, t}^{*}, i=1, \ldots, m$ provide optimal smooth functions of the following problem for any given $t \in[0, T]$ :

$$
\min _{h_{i, t} \in \mathcal{S}} \tilde{\mathbb{E}}\left[\left\{G_{t}-\sum_{i=1}^{m} h_{i, t}\left(S_{i, t}\right)\right\}^{2}\right],
$$

i.e., we would like to know if the values of options defined in (37) take optimal trajectories for any $t \in[0, T]$ to minimize the mean square error of (38). The following lemma plays an important role to answer this question:

Lemma 2 For each $i \in[1, m]$, let $\mathcal{F}_{i, t}, t \in[0, T]$ be the filtration generated by the single Brownian motion $W_{i, t}$, i.e., $\mathcal{F}_{i, t}$ is the filtration related to $\left\{S_{i, u}\right\}_{u \in[0, t]}$ only. Then, for any given measurable function $\psi$, it holds that

$$
\tilde{\mathbb{E}}\left[\psi\left(S_{1, T}, \ldots, S_{m, T}\right) \mid \mathcal{F}_{i, T}\right]=\tilde{\mathbb{E}}\left[\psi\left(S_{1, T}, \ldots, S_{m, T}\right) \mid S_{i, T}\right], \quad i=1, \ldots, m .
$$

Proof: We will show that condition (39) holds with $i=1$. Note that the same argument may be applied for $i \geq 2$, although we omit to explain the detail for brevity.

Consider the equivalent representation (27) for $S_{i, t}, i=1, \ldots, m_{0}$ based on the independent Brownian motions, $B_{1, t}, \ldots, B_{m, t}$, where $B_{1, t} \equiv W_{1, t}$. In this case, the left hand side of (39) may be rewritten as

$$
\tilde{\mathbb{E}}\left[\psi\left(S_{1, T}, \ldots, S_{m, T}\right) \mid \mathcal{F}_{1, T}\right]=\tilde{\mathbb{E}}\left[\psi_{1}\left(W_{1, T}, B_{2, T}, \ldots, B_{m, T}\right) \mid \mathcal{F}_{1, T}\right]
$$

using some function $\psi_{1}$. Since $\mathcal{F}_{1, t}$ is the filtration generated by $W_{1, t}$ and the other factors are independent of $W_{1, t}$, i.e., $W_{1, T}$ is $\mathcal{F}_{1, T}$-measurable and $B_{2, T}, \ldots, B_{m, T}$ are independent of $\mathcal{F}_{1, T}$, we can apply the Independence Lemma that a function $\hat{\psi}_{1}$ given as

$$
\hat{\psi}_{1}\left(w_{1}\right):=\mathbb{E}\left[\psi_{1}\left(w_{1}, B_{2, T}, \ldots, B_{m, T}\right)\right]
$$

satisfies the following condition:

$$
\hat{\psi}_{1}\left(W_{1, T}\right)=\mathbb{E}\left[\psi_{1}\left(W_{1, T}, B_{2, T}, \ldots, B_{m, T}\right) \mid \mathcal{F}_{1, T}\right] .
$$

Therefore, we can write the left hand side of (40) as

$$
\tilde{\mathbb{E}}\left[\psi\left(S_{1, T}, \ldots, S_{m, T}\right) \mid \mathcal{F}_{i, T}\right]=\hat{\psi}_{1}\left(W_{1, T}\right)
$$

By taking the conditional expectation given $S_{1, T}$ for both sides of (41), we have

$$
\begin{aligned}
& \tilde{\mathbb{E}}\left[\tilde{\mathbb{E}}\left[\psi\left(S_{1, T}, \ldots, S_{m, T}\right) \mid \mathcal{F}_{1, T}\right] \mid S_{1, T}\right]=\tilde{\mathbb{E}}\left[\hat{\psi}_{1}\left(W_{1, T}\right) \mid S_{1, T}\right] \\
\Rightarrow \quad & \tilde{\mathbb{E}}\left[\psi\left(S_{1, T}, \ldots, S_{m, T}\right) \mid S_{1, T}\right]=\hat{\psi}_{1}\left(W_{1, T}\right) .
\end{aligned}
$$

Conditions (41) and (42) indicates that (39) holds with $i=1$. Similarly, we can show that (39) holds for $i \geq 2$.

We are now in a position to show the following theorem for the solution to problem (38):

Theorem 2 For any given $t \in[0, T]$, the smooth functions $h_{i, t}^{*}, i=1, \ldots, m$ of (37) provide minimizers for the problem (38).

Proof: Because $f_{i}^{*}, i=1, \ldots, m$ are optimal smooth functions of the problem (32), the following equations are satisfied:

$$
\tilde{\mathbb{E}}\left[G_{T} \mid S_{i, T}\right]-\sum_{j=1}^{m} \tilde{\mathbb{E}}\left[f_{j}^{*}\left(S_{j, T}\right) \mid S_{i, T}\right]=0, \quad i=1, \ldots, m .
$$


Then, condition (43) may be rewritten as

$$
\tilde{\mathbb{E}}\left[G_{T} \mid \mathcal{F}_{i, T}\right]-\sum_{j=1}^{m} \tilde{\mathbb{E}}\left[f_{j}^{*}\left(S_{j, T}\right) \mid \mathcal{F}_{i, T}\right]=0, \quad i=1, \ldots, m
$$

by using Lemma 2.

From the tower property, we have

$$
\begin{aligned}
& \tilde{\mathbb{E}}\left[\tilde{\mathbb{E}}\left[G_{T} \mid \mathcal{F}_{i, T}\right] \mid S_{i, t}\right]-\sum_{j=1}^{m} \tilde{\mathbb{E}}\left[\tilde{\mathbb{E}}\left[f_{j}^{*}\left(S_{j, T}\right) \mid \mathcal{F}_{i, T}\right] \mid S_{i, t}\right]=0 \\
\Rightarrow & \tilde{\mathbb{E}}\left[G_{T} \mid S_{i, t}\right]-\sum_{j=1}^{m} \tilde{\mathbb{E}}\left[f_{j}^{*}\left(S_{j, T}\right) \mid S_{i, t}\right]=0 \\
\Rightarrow & \tilde{\mathbb{E}}\left[\tilde{\mathbb{E}}\left[G_{T} \mid \mathcal{F}_{t}\right] \mid S_{i, t}\right]-\sum_{j=1}^{m} \tilde{\mathbb{E}}\left[\tilde{\mathbb{E}}\left[f_{j}^{*}\left(S_{j, T}\right) \mid \mathcal{F}_{t}\right] \mid S_{i, t}\right]=0 \\
\Rightarrow & \tilde{\mathbb{E}}\left[G_{t} \mid S_{i, t}\right]-\sum_{j=1}^{m} \tilde{\mathbb{E}}\left[\tilde{\mathbb{E}}\left[f_{j}^{*}\left(S_{j, T}\right) \mid \mathcal{F}_{t}\right] \mid S_{i, t}\right]=0 .
\end{aligned}
$$

Noting that

$$
\begin{aligned}
\tilde{\mathbb{E}}\left[f_{j}^{*}\left(S_{j, T}\right) \mid \mathcal{F}_{t}\right] & =\tilde{\mathbb{E}}\left[f_{j}^{*}\left(S_{j, T}\right) \mid S_{j, t}\right] \\
& =h_{j, t}^{*}\left(S_{j, t}\right)=V_{j, t}, \quad j=1, \ldots, m
\end{aligned}
$$

from the Markov property, we finally obtain

$$
\tilde{\mathbb{E}}\left[G_{t} \mid S_{i, t}\right]-\sum_{j=1}^{m} \tilde{\mathbb{E}}\left[h_{j, t}^{*}\left(S_{j, t}\right) \mid S_{i, t}\right]=0, \quad i=1, \ldots, m
$$

implying that $h_{j, t}^{*}, i=1, \ldots, m$ in (37) provide optimal smooth functions of the problem (38). This completes the proof.

Theorem 2 indicates that, if we set the optimal smooth functions, $f_{i}^{*}, i=1, \ldots, m$, as payoff functions of options, the value of each individual option defined by (37) takes the optimal trajectory in the sense of minimum mean square error of the problem (38). This fact also suggests that there exists a self-financing portfolio whose value lies on the same optimal trajectory, i.e., there is a replicating portfolio for $V_{i, t}$ by dynamically trading $S_{i, t}, t \in[0, T]$ and the risk free asset.

To explain it more clearly, let $t=0$ and consider to construct a set of self-financing portfolios to replicate the terminal payoffs $f_{i}^{*}\left(S_{i, T}\right), i=1, \ldots, m$, where the initial values of the replicating portfolios are given by $V_{i, 0}, i=1, \ldots, m$ due to the no-arbitrage condition. From condition (33),

$$
G_{0}=\sum_{i=1}^{m} V_{i, 0}
$$

holds, and hence, we see that the mean square error from the basket option value is minimized with the sum of initial portfolio values at $t=0$. For the terminal values at $t=T$, the sum of payoffs determined by $f_{i}^{*}\left(S_{i, T}\right), i=1, \ldots, m$ minimizes the mean square error of the problem (32), and each payoff is replicated by the self-financing portfolio. Although it is not necessarily clear if we can obtain the same property in the meantime $t \in(0, T)$, Theorem 2 guarantees that the values of individual options and their replicating portfolios still take optimal trajectories in the sense of minimum mean square error under $\tilde{\mathbb{P}}$. 


\section{$5 \quad$ Further discussions}

In this section, we further discuss theoretical properties of optimal smooth functions for European type basket call/put options and their sensitivity analysis.

\subsection{Put-call parity for optimal smooth functions}

Let $C_{T}$ and $P_{T}$ be payoffs of European type basket call and put options given by

$$
C_{T}:=\left(\sum_{i=1}^{m} \alpha_{i} S_{i, T}-K\right)^{+}, \quad P_{T}:=\left(K-\sum_{i=1}^{m} \alpha_{i} S_{i, T}\right)^{+},
$$

where $K$ is a strike price, and consider the following problems for the call option,

$$
\min _{f_{i} \in \mathcal{S}} \mathbb{E}\left[\left\{C_{T}-\sum_{i=1}^{m} f_{i}\left(S_{i, T}\right)\right\}^{2}\right]
$$

and for the put option,

$$
\min _{h_{i} \in \mathcal{S}} \mathbb{E}\left[\left\{P_{T}-\sum_{i=1}^{m} h_{i}\left(S_{i, T}\right)\right\}^{2}\right] .
$$

For the optimal objective functions of these problems, we can obtain the following property that we state as a theorem:

Theorem 3 For the objective functions in (49) and (50), it holds that

$$
\min _{f_{i} \in \mathcal{S}} \mathbb{E}\left[\left\{C_{T}-\sum_{i=1}^{m} f_{i}\left(S_{i, T}\right)\right\}^{2}\right]=\min _{h_{i} \in \mathcal{S}} \mathbb{E}\left[\left\{P_{T}-\sum_{i=1}^{m} h_{i}\left(S_{i, T}\right)\right\}^{2}\right],
$$

i.e., both the objective functions achieve the same optimal level.

Proof: From the put-call parity, we have

$$
C_{T}=P_{T}+Y_{T}-K
$$

and the problem (49) may be rewritten as

$$
\begin{aligned}
\min _{f_{i} \in \mathcal{S}} \mathbb{E}\left[\left\{C_{T}-\sum_{i=1}^{m} f_{i}\left(S_{i, T}\right)\right\}^{2}\right] & =\min _{f_{i} \in \mathcal{S}} \mathbb{E}\left[\left\{P_{T}+Y_{T}-K-\sum_{i=1}^{m} f_{i}\left(S_{i, T}\right)\right\}^{2}\right] \\
& =\min _{f_{i} \in \mathcal{S}} \mathbb{E}\left[\left\{P_{T}-\sum_{i=1}^{m}\left(f_{i}\left(S_{i, T}\right)-\alpha_{i} S_{i, T}+\frac{K}{m}\right)\right\}^{2}\right] .
\end{aligned}
$$

Noting that

$$
f_{i}\left(S_{i, T}\right)-\alpha_{i} S_{i, T}+\frac{K}{m}
$$

is a smooth function of $S_{i, T}$ whereas the objective function in (50) is minimized over any smooth functions, we have

$$
\min _{f_{i} \in \mathcal{S}} \mathbb{E}\left[\left\{P_{T}-\sum_{i=1}^{m}\left(f_{i}\left(S_{i, T}\right)-\alpha_{i} S_{i, T}+\frac{K}{m}\right)\right\}^{2}\right] \geq \min _{h_{i} \in \mathcal{S}} \mathbb{E}\left[\left\{P_{T}-\sum_{i=1}^{m} h_{i}\left(S_{i, T}\right)\right\}^{2}\right] .
$$


Consequently,

$$
\min _{f_{i} \in \mathcal{S}} \mathbb{E}\left[\left\{C_{T}-\sum_{i=1}^{m} f_{i}\left(S_{i, T}\right)\right\}^{2}\right] \geq \min _{h_{i} \in \mathcal{S}} \mathbb{E}\left[\left\{P_{T}-\sum_{i=1}^{m} h_{i}\left(S_{i, T}\right)\right\}^{2}\right]
$$

holds.

On the other hand, we can use the relation

$$
P_{T}=C_{T}-Y_{T}+K
$$

to show that

$$
\min _{h_{i} \in \mathcal{S}} \mathbb{E}\left[\left\{P_{T}-\sum_{i=1}^{m} h_{i}\left(S_{i, T}\right)\right\}^{2}\right] \geq \min _{f_{i} \in \mathcal{S}} \mathbb{E}\left[\left\{C_{T}-\sum_{i=1}^{m} f_{i}\left(S_{i, T}\right)\right\}^{2}\right] .
$$

Conditions (52) and (53) indicate that (51) holds.

Theorem 3 implies that, once optimal smooth functions for the call option are found, we can construct optimal smooth functions for the put option, and vice versa. To see this, let $f_{1}^{*}, \ldots, f_{m}^{*}$ be optimal smooth functions for the problem (49) of call option. Then, defining $h_{i}^{*}$ by

$$
h_{i}^{*}\left(S_{i, T}\right):=f_{i}^{*}\left(S_{i, T}\right)-\alpha_{i} S_{i, T}+\frac{K}{m}, \quad i=1, \ldots, m
$$

yields

$$
\mathbb{E}\left[\left\{C_{T}-\sum_{i=1}^{m} f_{i}^{*}\left(S_{i, T}\right)\right\}^{2}\right]=\mathbb{E}\left[\left\{P_{T}-\sum_{i=1}^{m} h_{i}^{*}\left(S_{i, T}\right)\right\}^{2}\right]
$$

i.e., the functions $h_{1}^{*}, \ldots, h_{m}^{*}$ in (54) provide optimizers for the problem (50) of put option.

There is another implication obtained from Theorem 3 as follows: For sufficiently large $K\left(\gg Y_{0}\right)$, the call option is deep out-of-the-money (OTM) and the payoff tends to be zero. In fact, if $K \rightarrow \infty$, then $C_{T} \rightarrow 0$ and the optimal objective function in (49) approaches to zero from the following relation:

$$
\mathbb{E}\left[C_{T}^{2}\right] \geq \min _{f_{i} \in \mathcal{S}} \mathbb{E}\left[\left\{C_{T}-\sum_{i=1}^{m} f_{i}\left(S_{i, T}\right)\right\}^{2}\right] \geq 0
$$

For $K\left(\gg Y_{0}\right)$, the put option becomes deep in-the-money (ITM), but the optimal objective function in (50) approaches to zero as $K \rightarrow \infty$ due to condition (51). On the other hand, since

$$
\mathbb{E}\left[P_{T}^{2}\right] \geq \min _{h_{i} \in \mathcal{S}} \mathbb{E}\left[\left\{P_{T}-\sum_{i=1}^{m} h_{i}\left(S_{i, T}\right)\right\}^{2}\right]=\min _{f_{i} \in \mathcal{S}} \mathbb{E}\left[\left\{C_{T}-\sum_{i=1}^{m} f_{i}\left(S_{i, T}\right)\right\}^{2}\right] \geq 0 .
$$

holds, the optimal objective functions in (49) and (50) approach to zero as $K \rightarrow 0$. In summary, both the optimal objective functions for call and put approach to zero when $K \rightarrow \infty$ or $K \rightarrow 0$. Such an observation may lead to a conjecture that the optimal objective function is maximized around at-the-money (ATM) case, i.e., in terms of the minimum mean square error, the ATM option is the most difficult to hedge as indicated by the usual options' theory that the option gamma may be maximized around the ATM case. We will confirm this based on the numerical experiment in Section 6 .

\subsection{Computation of Greeks and sensitivity analysis}

Since payoff functions are non-differentiable for standard calls and puts, it is usually difficult to compute Greeks such as delta or gamma. For example, when $G_{T}$ is defined as in (24), the computation of delta on asset $i$ may 
involve $^{3}$

$$
\frac{\partial \tilde{\mathbb{E}}\left[G_{T}\right]}{\partial S_{i, 0}},
$$

where $\tilde{\mathbb{E}}$ is the expectation under the corresponding risk neutral probability measure. Since $G_{T}$ depends on the terminal prices of $m_{0}$ assets, $S_{1, T}, \ldots, S_{m_{0}, T}, \tilde{\mathbb{E}}\left[G_{T}\right]$ may require multiple integration and is difficult to compute even numerically. Although we might be able to apply the Monte Carlo method to compute $\tilde{\mathbb{E}}\left[G_{T}\right]$, the numerical differentiation of $\tilde{\mathbb{E}}\left[G_{T}\right]$ combined with Monte Carlo seems to be unrealistic.

On the other hand, in the case of smooth functions, delta may be computed efficiently. To see this, let $f$ be a smooth function, and consider the following partial derivative:

$$
\frac{\partial \tilde{\mathbb{E}}\left[f\left(S_{i, T}\right)\right]}{\partial S_{i, 0}} .
$$

If $f=f_{i}^{*}$, (59) provides delta on $S_{i, 0}$ when approximating the payoff of basket option using optimal smooth functions, $f_{i}^{*}, i=1, \ldots, m$. Assume that the price dynamics of $S_{i, t}$ is given as in (12), i.e., $S_{i, t}$ satisfies

$$
S_{i, t}=S_{i, 0} \exp \left[\nu_{i} t+\sigma_{i} W_{i, t}\right] .
$$

Let $h(x, y)$ be a function of $(x, y)$ defined by

$$
h(x, y):=f\left(x \exp \left[\nu_{i} T+\sigma_{i} y\right]\right)
$$

and rewrite (59) as

$$
\frac{\partial \tilde{\mathbb{E}}\left[h\left(S_{i, 0}, W_{i, T}\right)\right]}{\partial S_{i, 0}} .
$$

Since $h$ is differentiable with respect to $(x, y)$, we can apply the Leibniz's rule to switch the integration and differentiation as

$$
\begin{aligned}
\frac{\partial \tilde{\mathbb{E}}\left[h\left(S_{i, 0}, W_{i, T}\right)\right]}{\partial S_{i, 0}} & =\tilde{\mathbb{E}}\left[\frac{\partial h\left(S_{i, 0}, W_{i, T}\right)}{\partial S_{i, 0}}\right] \\
& =\tilde{\mathbb{E}}\left[\frac{\partial f\left(S_{i, T}\right)}{\partial S_{i, 0}}\right] .
\end{aligned}
$$

Condition (61) may be written as

$$
\begin{aligned}
\tilde{\mathbb{E}}\left[\frac{\partial f\left(S_{i, T}\right)}{\partial S_{i, 0}}\right] & =\tilde{\mathbb{E}}\left[f^{\prime}\left(S_{i, T}\right) \frac{\partial S_{i, T}}{\partial S_{i, 0}}\right] \\
& =\tilde{\mathbb{E}}\left[f^{\prime}\left(S_{i, T}\right) \exp \left(\nu_{i} T+\sigma_{i} W_{i, T}\right)\right] .
\end{aligned}
$$

where $f^{\prime}(x)$ stands for the first order derivative of $f(x)$. In the case of cubic splines in $(11), f^{\prime}(x)$ is given as

$$
f^{\prime}(x)=c_{1}+\frac{1}{4} \sum_{k=1}^{N} \theta_{k}\left(x-x_{i}^{(k)}\right)^{2} \operatorname{sign}\left(x-x_{i}^{(k)}\right)
$$

where $\operatorname{sign}(\cdot)$ is the sign function. Therefore, we see that the delta in (59) may be computed efficiently using the common technique such as the standard finite difference method (Hull and White (1990)). Similarly, we can compute other Greeks efficiently.

Computational tractability for delta is not only important for sensitivity analysis but also for constructing replicating portfolio. For hedging basket options by trading the underlying stocks, a standard approach is to

\footnotetext{
${ }^{3}$ We omit the discount factor for brevity.
} 
try to work on the formula (58), but this approach can not be used in practice due to the reasons described at the beginning of this subsection. Another approach is to approximate the payoff by smooth functions using our proposed methodology and compute delta using (59) with $f=f_{i}^{*}$, i.e.,

$$
\frac{\partial \tilde{\mathbb{E}}\left[f_{i}^{*}\left(S_{i, T}\right)\right]}{\partial S_{i, 0}}, \quad i=1, \ldots, m
$$

It should be mentioned that each delta in (64) can be computed separately from the other variables $S_{j, t}, j \neq i$. This fact may be the most advantage over the original delta in (58), i.e., we can compute delta in (64) off-line using the two dimensional finite difference method (Hull and White (1990)). Off course there is a gap between the payoff of basket option and the sum of smooth functions, our numerical experiment in Section 6 suggests that this gap is not significant on average.

\section{Numerical experiment}

\subsection{Case 1: Hedging market index using several stocks}

In this numerical experiment, we first consider a problem of hedging an option whose underlying is a market index (being nontraded) using several stocks, where each asset dynamics is modeled as a geometric Brownian motion as in (12) and (13). We will formulate the minimum variance hedging problem and solve it by applying the proposed methodology.

We use the empirical data from the Tokyo Stock Exchange (TSE) in the period of 2003-2005 for estimating the volatility and correlation parameters of stock returns, where the market index is assumed to be TOPIX and five stocks, $S_{1}, \ldots, S_{5}$, are chosen from those listed in the TSE. The correlation and volatility parameters of stock returns are estimated as in Table 1, whereas we assume that each expected stock return corresponding to the drift parameter has the same sharp ratio $(=0.25)$ with risk free interest rate $r=0.05$.

Table 1: Volatility and correlation of the stock returns with drift having the same sharp ratio $(=0.25)$

\begin{tabular}{c||llllll}
\hline & INDEX & $S_{1}$ & $S_{2}$ & $S_{3}$ & $S_{4}$ & $S_{5}$ \\
\hline INDEX & 1 & & & & & \\
$S_{1}$ & 0.552 & 1 & & & & \\
$S_{2}$ & 0.636 & 0.298 & 1 & & & \\
$S_{3}$ & 0.615 & 0.476 & 0.346 & 1 & & \\
$S_{4}$ & 0.557 & 0.291 & 0.406 & 0.341 & 1 & \\
$S_{5}$ & 0.604 & 0.315 & 0.457 & 0.287 & 0.389 & 1 \\
\hline Volatility & 0.176 & 0.549 & 0.227 & 0.421 & 0.307 & 0.232 \\
\hline Drift & 0.094 & 0.187 & 0.107 & 0.155 & 0.127 & 0.108 \\
\hline
\end{tabular}

We solve the problem (1) to find the minimizers $f_{1}^{*}, \ldots, f_{5}^{*}$ for hedging an at-the-money European call option with maturity $T=1 / 4$, where the initial prices (or initial values) are set to be $Y_{0}=100$ and $S_{i, 0}=100, i=$ $1, \ldots, 5$. Figure 1 illustrates the minimizers, where the thin line refers to $S_{1, T}$ vs. $f_{1}^{*}\left(S_{1, T}\right)$, the solid line to $S_{2, T}$ vs. $f_{2}^{*}\left(S_{2, T}\right)$, the broken line to $S_{3, T}$ vs. $f_{3}^{*}\left(S_{3, T}\right)$, the dashed line to $S_{4, T}$ vs. $f_{4}^{*}\left(S_{4, T}\right)$, and the dotted line to $S_{5, T}$ vs. $f_{5}^{*}\left(S_{5, T}\right)$.

The correlation coefficient between $G_{T}$ and $\sum_{i=1}^{5} f_{i}^{*}\left(S_{i, T}\right)$ may provide a hedge effect, which is computed as 0.805 . We see that a high hedge effect is obtained in this numerical experiment. 


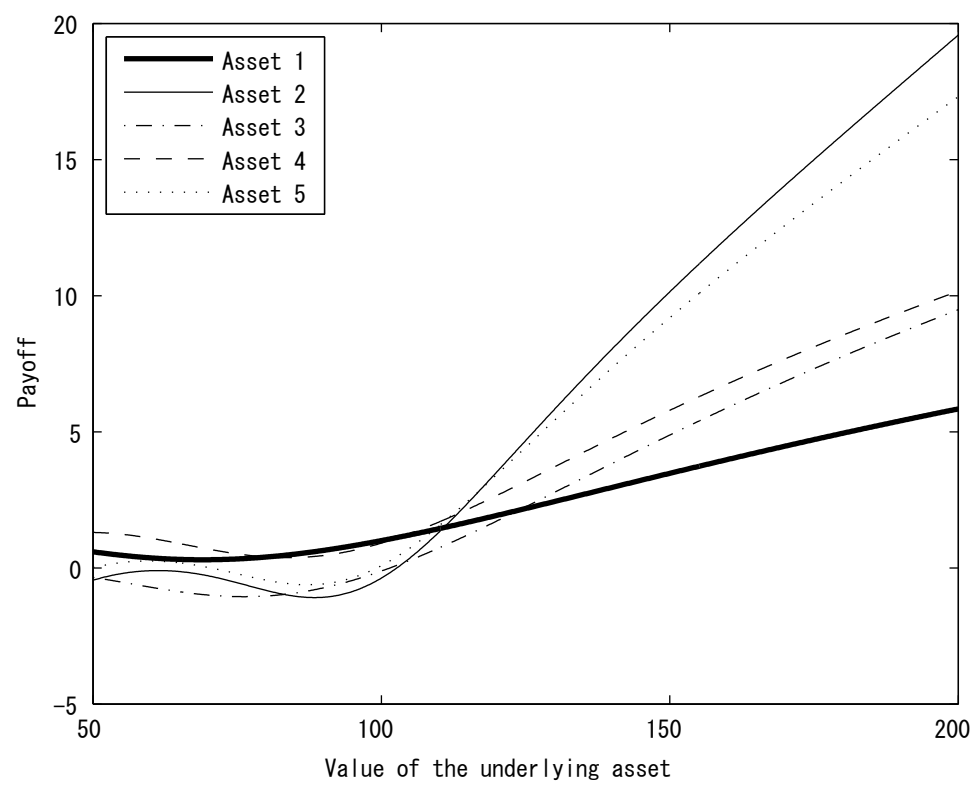

Figure 1: Optimal smooth functions for the minimum variance

\subsection{Case 2: Hedging basket options}

Next, we consider the problems of hedging basket call/put options described in (49) and (50). Assume that the payoff depends on the weighted sum of five stocks, $S_{1}, \ldots, S_{5}$, having the same parameter values in Table 1 . To investigate the effect of moneyness (ATM, OTM, or ITM) more explicitely, we assume that the mean rate of return for each asset and the risk free rate are zero, i.e., $\mu_{i}=0, i=1, \ldots, m$ and $r=0$. We set $S_{i, 0}=100$ and $\alpha_{i}=1 / 5, i=1, \ldots, 5$ so that the initial value of the underlying of basket option is given by $\sum_{i=1}^{5} \alpha_{i} S_{i, 0}=100$.

At first, we solve the minimum variance hedging problems for different values of strike prices and estimate their hedge errors. Here we generated 5 dimensional random numbers from independent normal distributions, and used the first 4 dimensions for computing each $\hat{g}_{i}\left(w_{i}\right), i=1, \ldots, m$ based on the formula in Theorem 1 . Then we simulated

$$
C_{T}-\sum_{i=1}^{5} f_{i}^{*}\left(S_{i, T}\right),
$$

and

$$
P_{T}-\sum_{i=1}^{5} h_{i}^{*}\left(S_{i, T}\right),
$$

for the optimal smooth functions $f_{i}^{*}, i=1, \ldots, m$ and $h_{i}^{*}, i=1, \ldots, m$, using all the outcomes of random numbers, where the number of samples for each dimension is $N=100,000$ in our simulation.

The left and the right hand sides of Figure 2 show the relation between the standard deviations of hedging errors vs. strike prices for the call and the put options, respectively. The vertical axis refers to the strike price divided by the initial price $(=100)$, whereas the horizontal axis to the standard deviations of hedging errors (65) in the case of call option and (66) in the case of put option. From these figures, we have two important observations: First, we see that both plots are exactly the same as indicated by Theorem 3, where the mean square errors (or the corresponding standard deviations) for the call and the put are equal. Second, 
this numerical result suggests that the mean square error is maximized around the ATM case, i.e., as far as the mean square error is concerned, the ATM option seems to be the most difficult to hedge on average.
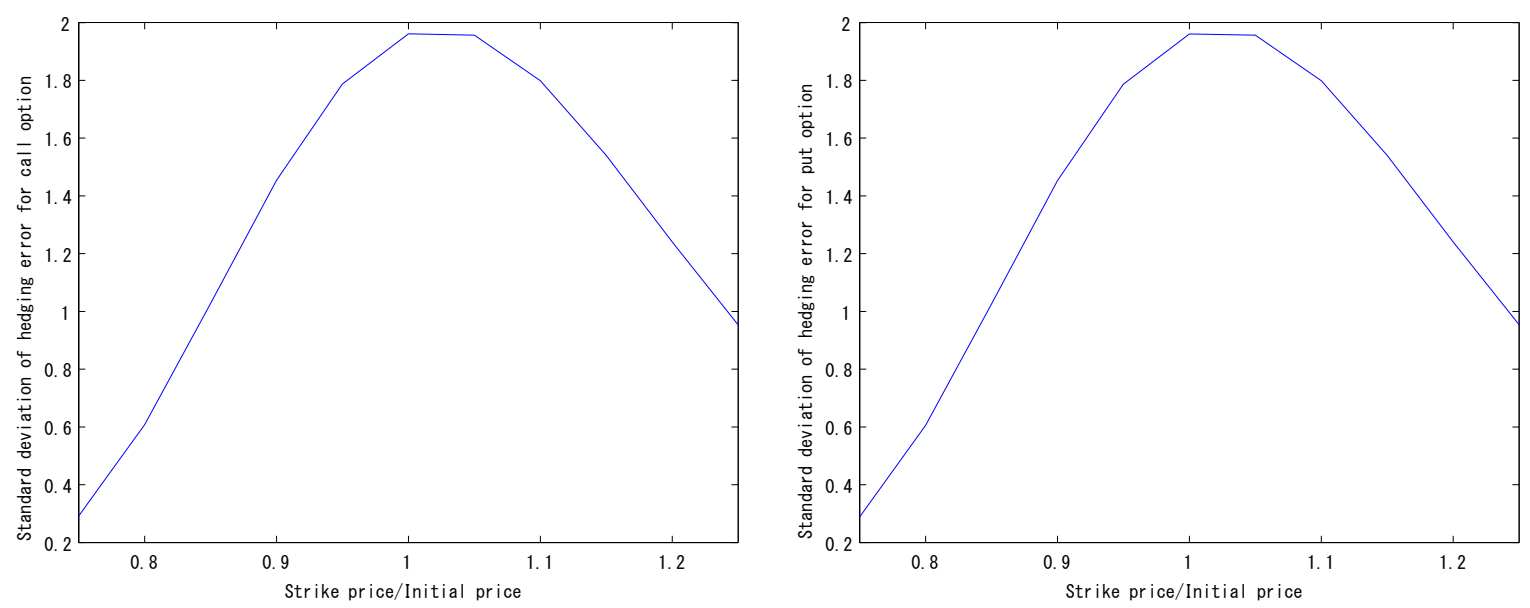

Figure 2: Standard deviations of hedging errors for call option (left) and put option (right)

The left hand side of Figure 3 shows the scatter plot of $C_{T}$ vs. $\sum_{i=1}^{5} f_{i}^{*}\left(S_{i, T}\right)$ for the ATM call option, whereas the right hand side that of $P_{T}$ vs. $\sum_{i=1}^{5} f_{i}^{*}\left(S_{i, T}\right)$ for the ATM put option. Similar to the first numerical experiment, we can evaluate the hedge effects by the correlation coefficients of $C_{T}$ vs. $\sum_{i=1}^{5} f_{i}^{*}\left(S_{i, T}\right)$ and $P_{T}$ vs. $\sum_{i=1}^{5} f_{i}^{*}\left(S_{i, T}\right)$, respectively for the call and the put options, and in this numerical experiment, they are computed as 0.969 and 0.955 . We see that the payoffs of the basket options may be approximated with high accuracy using those of individual options.
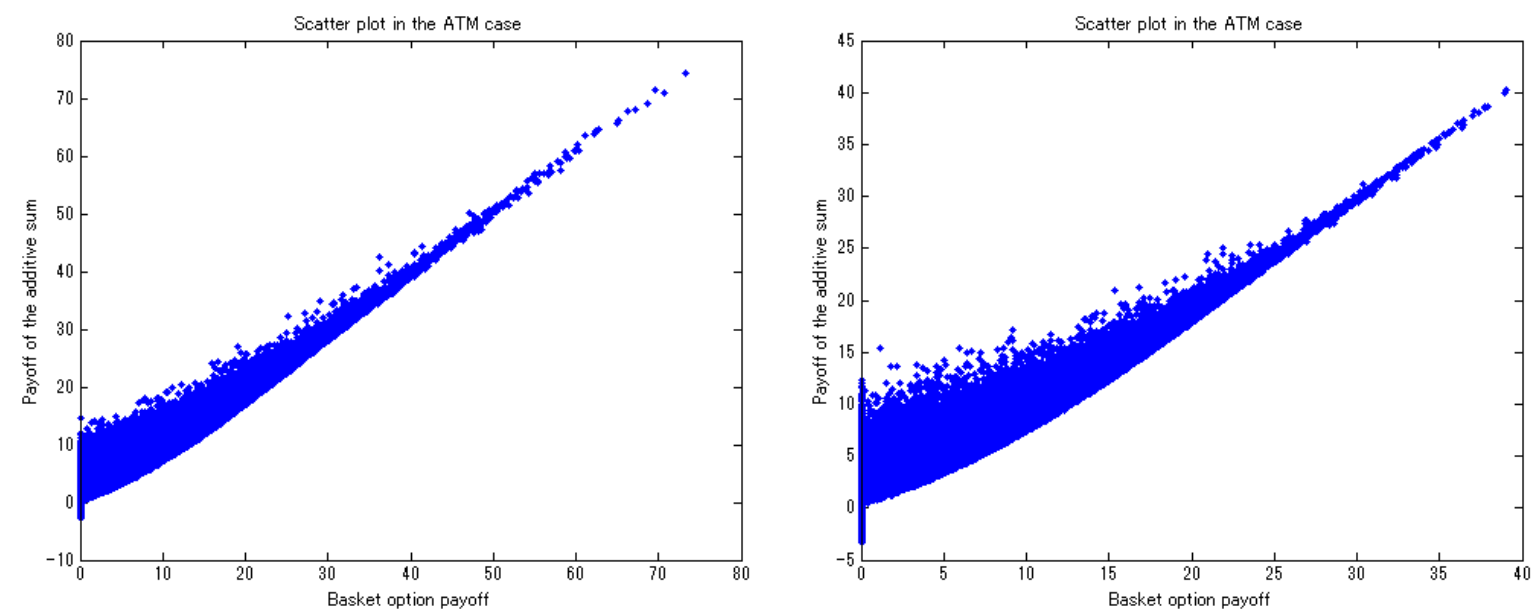

Figure 3: Scatter plots for the ATM call option (left) and the ATM put option (right)

\subsection{Case 3: Comparison with super-hedging strategy}

Finally, we compare our methodology with a super-hedging strategy in $\mathrm{Su}$ (2008), which provides a tighter upper bound than usual Jensen's inequality by optimizing a set of parameters related to strike prices of individual 
options. The basic idea is the same as the earlier work of Hobson et al. (2005) that bases on the following relation in the case of basket call option:

$$
\begin{aligned}
C_{T}=\left(\sum_{i=1}^{m} \alpha_{i} S_{i, T}-K\right)^{+} & =\left[\sum_{i=1}^{m} \alpha_{i}\left(S_{i, T}-\frac{\beta_{i}}{\alpha_{i}} K\right)\right]^{+} \\
& \leq \sum_{i=1}^{m} \alpha_{i}\left(S_{i, T}-\frac{\beta_{i}}{\alpha_{i}} K\right)^{+}, \sum_{i=1}^{m} \beta_{i}=1
\end{aligned}
$$

The least upper bound problem on $C_{0}=e^{-r T} \tilde{\mathbb{E}}\left[C_{T}\right]$, providing the minimum cost of super-hedging, is then formulated as

$$
\bar{C}_{0}:=\min _{\beta_{1}, \ldots, \beta_{m}}\left\{\sum_{i=1}^{m} \alpha_{i} e^{-r T} \tilde{\mathbb{E}}\left[\left(S_{i, T}-\frac{\beta_{i}}{\alpha_{i}} K\right)^{+}\right] \mid \sum_{i=1}^{m} \beta_{i}=1\right\} .
$$

On the other hand, by defining $\bar{\alpha}$ and $\varphi(x)$ as

$$
\bar{\alpha}:=\sum_{i=1}^{m} \alpha_{i}, \quad \varphi(x):=\left[\bar{\alpha}\left(x-\frac{K}{\bar{\alpha}}\right)\right]^{+},
$$

an upper bound from Jensen's inequality may be derived from the following relation:

$$
C_{T}=\varphi\left(\sum_{i=1}^{m} \frac{\alpha_{i}}{\bar{\alpha}} S_{i, T}\right) \leq \sum_{i=1}^{m} \frac{\alpha_{i}}{\bar{\alpha}} \varphi\left(S_{i, T}\right)=\sum_{i=1}^{m} \alpha_{i}\left(S_{i, T}-\frac{K}{\bar{\alpha}}\right)^{+} .
$$

Clearly, the upper bound given by Jensen's inequality, denoted by

$$
\bar{C}_{0}^{J}:=\sum_{i=1}^{m} \alpha_{i} e^{-r T} \tilde{\mathbb{E}}\left[\left(S_{i, T}-\frac{K}{\bar{\alpha}}\right)^{+}\right] .
$$

is no less than $\bar{C}_{0}$, since $\bar{C}_{0}^{J}$ is a special case with $\beta_{i}=\alpha_{i} / \bar{\alpha}, i=1, \ldots, m$ in the objective function of (68). Note that $\beta_{i}, i=1, \ldots, m$ minimizing the objective function in (68) may be found numerically by constructing a Lagrange function as shown in Su (2008).

When applying the super-hedging strategy, one may sell the basket option at $t=0$ and buy a set of call options on individual assets so that the initial cash flow is given as

$$
C_{0}-\bar{C}_{0}
$$

Since the terminal cash flow at $t=T$ from this position is

$$
\sum_{i=1}^{m} \alpha_{i}\left(S_{i, T}-\frac{\bar{\beta}_{i}}{\alpha_{i}} K\right)^{+}-C_{T}
$$

the terminal hedging error, denoted by $H_{e}$, may be given as

$$
H_{e}:=\sum_{i=1}^{m} \alpha_{i}\left(S_{i, T}-\frac{\bar{\beta}_{i}}{\alpha_{i}} K\right)^{+}-C_{T}+e^{r T}\left(C_{0}-\bar{C}_{0}\right)
$$

where the risk free interest rate is compounded to the initial cost to adjust the present and the future values.

Here we compare the hedging errors between the minimum variance hedging strategy (or "optimal hedging" for short) and the super-hedging strategy (or "super-hedging" for short) based on the same problem setting as that in Subsection 6.2. Since we have assumed that $\mu_{i}=0, i=1, \ldots, m$ and $r=0$, the physical probability measure provides the risk neutral probability measure, and it holds that

$$
\mathbb{E}\left(H_{e}\right)=\tilde{\mathbb{E}}\left(H_{e}\right)=0 .
$$


For optimal hedging, note the expected values of hedging errors are also zero for both under the risk and physical probability measures. Therefore, the standard deviation of hedging error may be thought of a measure of risk under the zero expected return.

Figure 4 compares the standard deviations of hedging errors for optimal hedging (solid line) and superhedging (dashed line) for different values of strikes. Similar to Figure 2, the vertical axis in each plot refers to the strike price divided by the initial price $(=100)$, whereas the horizontal axis to the standard deviation of hedging error. The top left plot is for $T=1 / 24$ (1/2 month maturity), the top right for $T=1 / 12$ ( 1 month maturity), the bottom left for $T=3 / 12$ (3 month maturity), and the bottom right for $T=6 / 12$ (6 month maturity). As expected, optimal hedging always provides a better hedge effect in terms of standard deviation, due to the fact that optimal hedging minimizes the mean square error (or the variance with zero expected value) directly. In particular, the difference is more emphasized with a larger strike price and a longer maturity.

To compare the worst case loss, we computed $95 \%$ Value-at-Risk (VaR) for the hedge errors and obtained Figure 5, where the solid line refers to $95 \%$ VaR of the hedging error from optimal hedging and the dashed to that from super-hedging. Similar to Figure 4, the top left plot is for $T=1 / 24$ (1/2 month maturity), the top
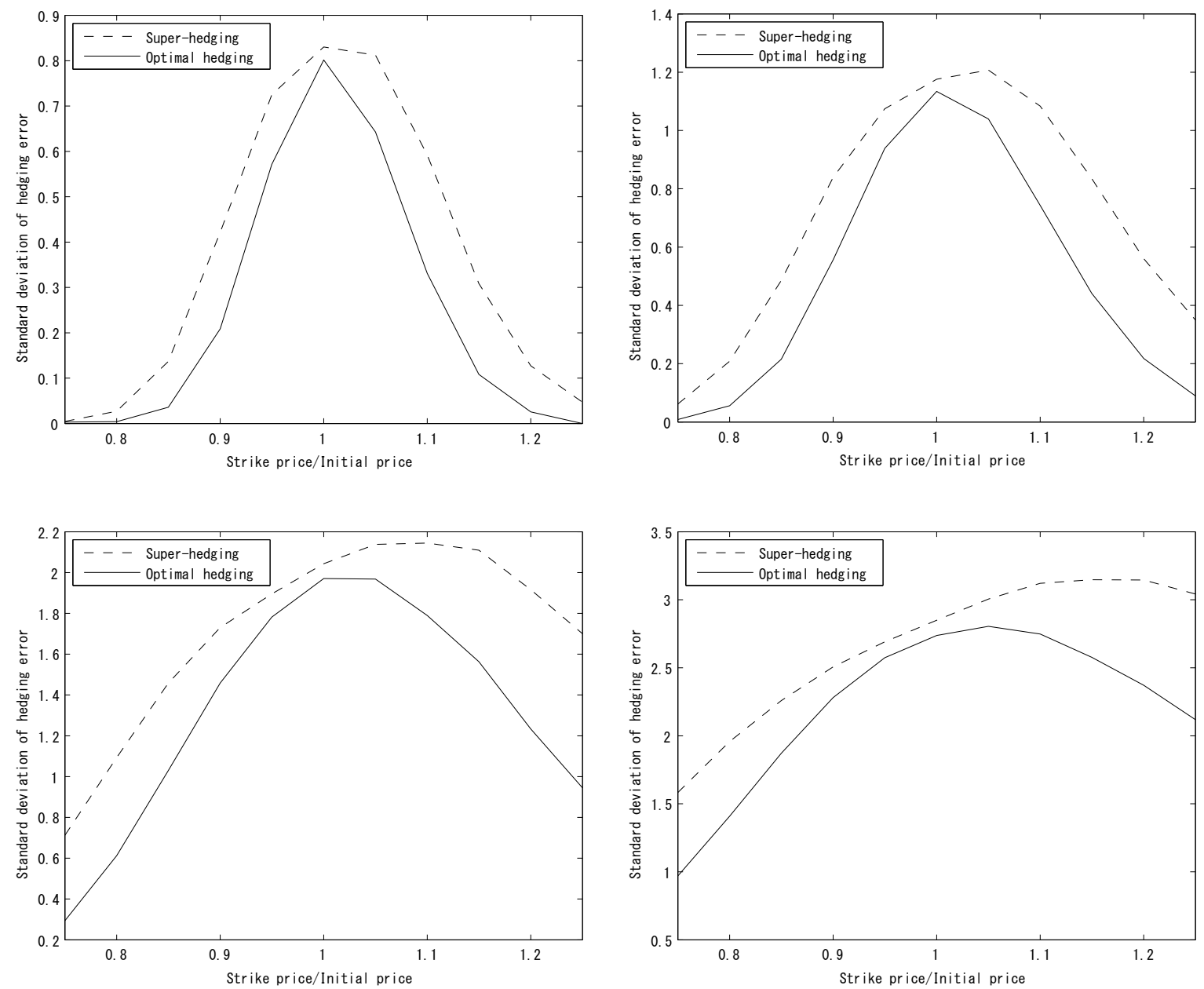

Figure 4: Standard deviations of hedging errors for optimal hedging (solid line) and super-hedging (dashed line): The top left plot is for $T=1 / 24$ (1/2 month maturity), the top right for $T=1 / 12$ ( 1 month maturity), the bottom left for $T=3 / 12$ (3 month maturity), and the bottom right for $T=6 / 12$ (6 month maturity). 
right for $T=1 / 12$ ( 1 month maturity), the bottom left for $T=3 / 12$ (3 month maturity), and the bottom right for $T=6 / 12$ (6 month maturity). In this case, super hedging tends to be better, in particular, when the strike price is within the range of $\pm 10-15 \%$ from the initial value of the underlying. On the other hand, for a shorter maturity in the deep OTM and ITM cases, optimal hedging tends to be better in terms of $95 \%$ VaR. In summary, we conclude that optimal hedging is always better if we take standard deviation as a performance measure of the hedge, whereas in terms of the worst case error, super-hedging tends to provide a better bound with a given confidence level, i.e., $95 \%$ VaR in this example.
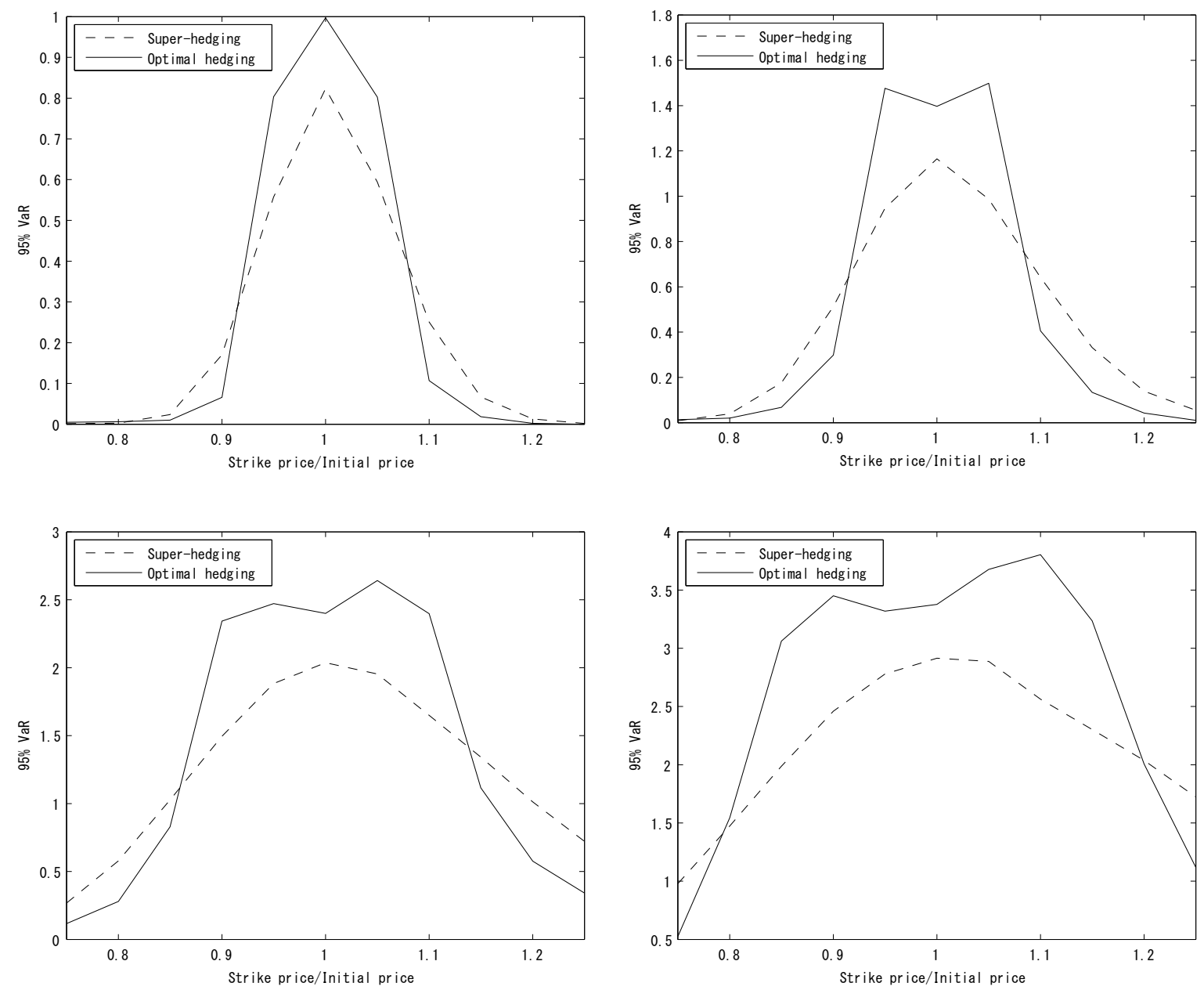

Figure 5: 95\% VaR of hedging errors for optimal hedging (solid line) and super-hedging (dashed line): The top left plot is for $T=1 / 24$ ( $1 / 2$ month maturity), the top right for $T=1 / 12$ ( 1 month maturity), the bottom left for $T=3 / 12$ (3 month maturity), and the bottom right for $T=6 / 12$ (6 month maturity).

Remark 2 Note that the super-hedging strategy is completely static and is constructed using individual options with standard payoff functions. Although the optimal hedging strategy uses options with any payoff functions and is more difficult to construct a static position, dynamic hedging strategy may be used to replicate each payoff of individual option as demonstrated in Section 5. It should be mentioned that the optimal hedging strategy may be applied for any payoff functions of the target derivative, whereas for applying the super-hedging strategy, the payoff functions are restricted to be convex because of Jensen's inequality. Using optimal hedging strategy, we 
can consider general payoff structure or general derivatives such as spread options, compound options, and some of exiotics including digital options.

\section{Conclusion}

In this paper, we have considered an optimal hedging problem, in which smooth functions of individual assets are searched over to minimize the mean square error from the payoff of multivariate derivative security. At first, we derived a set of linear equations to construct optimal smooth functions by applying a necessary and sufficient condition for optimality and suitable discretization. It was shown that the computations involving conditional expectations for multivariate derivatives may be reduced to those of unconditional expectations and that the total procedure can be executed efficiently. We investigated the theoretical properties for the optimal smooth functions and clarified the following three facts: (i) the value of each individual option takes an optimal trajectory to minimize the mean square hedging error under the risk neutral probability measure, (ii) optimal smooth functions for the put option may be constructed using those for the call option (and vice versa), and (iii) delta in the replicating portfolio for multivariate option may be computed efficiently. Finally, we demonstrated numerical experiments to show the effectiveness of our proposed methodology, where we compared our proposed methodology with the super-hedging strategy for basket options. Based on the numerical experiment, we verified that the optimal hedging strategy is better if we take standard deviation as a performance measure of the hedge, whereas in terms of the worst case error, super-hedging tends to provide a better bound with a given confidence level.

\section{References}

[1] J. Alcock and P. Gray (2005), "Dynamic, nonparametric hedging of European style contingent claims using canonical valuation," Finance Research Letters, 2, pp. 41-50.

[2] Y. Ait-Sahalia and A.W. Lo (1998), "Nonparametric Estimation of State-Price Densities Implicit in Financial Asset Prices," Journal of Finance, 52, pp. 499-548.

[3] F. Black and M. Scholes (1973), "The Pricing of Options and Corporate Liabilities," Journal of Political Economy, 81, 637-654.

[4] M. Broadie, J. Detemple, E. Ghysels and O. Torres (2000), "Nonparametric estimation of American options' exercise boundaries and call prices," Journal of Economic Dynamics and Control, 24, 1829-1857.

[5] P. Carr and D. Madan (2001), "Optimal positioning in derivative securities," Journal of Financial Econometrics, 1(3), 327-364.

[6] T. Daglish (2003), "A Pricing and Hedging Comparison of Parametric and Nonparametric Approaches for American Index Options," Quantitative Finance, 1, 19-37.

[7] D. Duffie and H.R. Richardson (1991), "Mean-variance hedging in continuous time," Annals Appl. Probability, 1, 1-15.

[8] R. Garciaa and R. Gencay (2000), "Pricing and hedging derivative securities with neural networks and a homogeneity hint," Journal of Econometrics, 94, pp. 93-115.

[9] P. Gray, S. Edwards and E. Kalotay (2007), "Canonical valuation and hedging of index options," Journal of Futures Markets, 27(8), pp. 771-790. 
[10] T. Hastie and R. Tibshirani (1990), Generalized Additive Models, Chapman \& Hall.

[11] D. Hobson, P. Laurencec and T.H. Wang (2005), "Static-Arbitrage Upper Bounds for the Prices of Basket Options," Quantitative Finance, 5(4), 329-342.

[12] J. Hull and A. White (1990), "Valuing Derivative Securities Using the Explicit Finite Difference Method," Journal of Financial and Quantitative Analysis, 25, pp. 87-100.

[13] J.M. Hutchinson, A.W. Lo and T. Poggio (1994), "A Nonparametric Approach to Pricing and Hedging Derivative Securities via Learning Networks," Journal of Finance, 49, pp. 851-889.

[14] R. Merton (1973), "Theory of rational option pricing," Bell Journal of Economics and Management Science, 4, pp. 141-184.

[15] J.A. Primbs (2009), "Dynamic hedging of basket options under proportional transaction costs using receding horizon control," International Journal of Control, 82(10), pp. 1841-1855.

[16] E.S. Schwartz and C. Tebaldi (2006), "Illiquid Assets and Optimal Portfolio Choice," NBER Working Paper No. 12633, (http://www.nber.org/papers/w12633).

[17] S.E. Shreve (2004), Stochastic Calculus for Finance II: Continuous-Time Models, Springer.

[18] M. Stutzer (1996), "A Simple Nonparametric Approach to Derivative Security Valuation," Journal of Finance, 51, pp. 1633-1652.

[19] X. Su (2008), "Essays on Basket Options Hedging and Irreversible Investment Valuation," Ph.D. Dissertation, University of Bonn.

[20] S.N. Wood (2006), Generalized Additive Models: An Introduction with R, Chapman \& Hall.

[21] Y. Yamada (2007), "Valuation and Hedging of Weather Derivatives on Monthly Average Temperature," Journal of Risk, 10(1), pp. 101-125.

[22] Y. Yamada (2008a), "Optimal design of wind derivatives based on prediction errors (in Japanese)," JAFEE journal, 7, pp. 152-181.

[23] Y. Yamada (2008b), "Optimal hedging of prediction errors using prediction errors," Asia-Pacific Financial Markets, 15(1), pp. 67-95. 